\title{
New concepts in white adipose tissue physiology
}

\author{
A.R.G. Proença ${ }^{3 *}$, R.A.L. Sertié ${ }^{1 *}$, A.C. Oliveira ${ }^{2 *}$, A.B. Campaña ${ }^{1}$, R.O. Caminhotto ${ }^{1}$ \\ P. Chimin ${ }^{1}$ and F.B. Lima $^{1}$ \\ ${ }^{1}$ Departamento de Fisiologia e Biofísica, Instituto de Ciências Biomédicas, Universidade de São Paulo, São Paulo, SP, Brasil \\ ${ }^{2}$ Instituto Superior de Ciências Biomédicas, Universidade Estadual do Ceará, Fortaleza, CE, Brasil \\ ${ }^{3}$ Laboratório de Biotecnologia, Faculdade de Ciências Aplicadas, Universidade Estadual de Campinas, Limeira, SP, Brasil
}

\begin{abstract}
Numerous studies address the physiology of adipose tissue (AT). The interest surrounding the physiology of AT is primarily the result of the epidemic outburst of obesity in various contemporary societies. Briefly, the two primary metabolic activities of white AT include lipogenesis and lipolysis. Throughout the last two decades, a new model of AT physiology has emerged. Although AT was considered to be primarily an abundant energy source, it is currently considered to be a prolific producer of biologically active substances, and, consequently, is now recognized as an endocrine organ. In addition to leptin, other biologically active substances secreted by AT, generally classified as cytokines, include adiponectin, interleukin-6, tumor necrosis factor-alpha, resistin, vaspin, visfatin, and many others now collectively referred to as adipokines. The secretion of such biologically active substances by AT indicates its importance as a metabolic regulator. Cell turnover of AT has also recently been investigated in terms of its biological role in adipogenesis. Consequently, the objective of this review is to provide a comprehensive critical review of the current literature concerning the metabolic (lipolysis, lipogenesis) and endocrine actions of AT.
\end{abstract}

Key words: Adipose tissue; Lipogenesis; Lipolysis; Adipogenesis; Endocrine

\section{Introduction}

It is well known that adipose tissue (AT) is the largest energy reservoir of the body (1). Due to a lack of precise knowledge regarding the functions of AT, for a long time its metabolic purpose was considered to be passive participation in the metabolic support system. Throughout the previous two decades, however, this notion has changed, and a new functional view of AT has emerged. AT is now known to be a producer of biologically active substances and is consequently recognized as an endocrine organ, thus indicating a role for AT in the regulation of energy metabolism (1).

Interest in the functional role of AT arose primarily due to the epidemic surge of obesity in various contemporary societies. This epidemic of obesity resulted in an increase in AT research triggered primarily by an influx of AT modus operandi, thus providing a better understanding of the role of AT in the body's metabolic control. Animal and human studies (both in vivo and in vitro), utilizing several methodological tools such as cellular and molecular physiological, pharmacological, and clinical setting approaches, have been utilized to investigate the functional role of AT. Such studies, in addition to clarifying the role of individual factors affecting metabolic control such as diet, exercise, disease, age, and stress, also increased understanding of the repercussions of an increase or a decrease in adiposity in the body as a whole.

There are two primary metabolic activities of white AT (1). They are lipogenesis (fatty acid synthesis and storage) and lipolysis (mobilization or hydrolysis of triglycerides). Both lipolysis and lipogenesis are regulated by the integration of endocrine and neural mechanisms, which cooperate in order to maintain the relative constancy of body fat under normal conditions.

The discovery of leptin, a hormone secreted by AT and related to satiety, significantly altered the perception of AT in the body's metabolism (2). Other biologically active substances, generally known as cytokines, including adiponectin, interleukin (IL)-6, tumor necrosis factor alpha $(\mathrm{TNF}-\alpha)$, resistin, vaspin, visfatin, and many others secreted by AT have been identified (now collectively

Correspondence: A.R.G. Proença, Laboratório de Biotecnologia, Faculdade de Ciências Aplicadas, UNICAMP, R. Pedro Zaccaria, 1300, 13484-350 Limeira, SP, Brasil. E-mail: aproenca19@hotmail.com

${ }^{*}$ These authors contributed equally to this work.

Received May 2, 2013. Accepted November 4, 2013. First published online February 18, 2014. 
termed adipokines), reinforcing the importance of AT as a metabolic regulator (3). Adipokines have many functions, including regulation of carbohydrate and lipid metabolism, regulation of feeding behavior (including hunger and satiety), and insulin sensitivity. The expression of adipokines is altered by many clinical conditions such as obesity and diabetes, indicating a role for adipokines in the metabolic control of such illnesses.

Cell turnover of AT has also been extensively studied. The fat tissue hyperplasia occurs ultimately due to a physiological imbalance between adipogenesis and apoptosis, which ultimately results in the gain or loss of fat mass. In pubescent adults, the formation of new fat cells is an active process, resulting in the growth of AT. Throughout pubescent adulthood, a certain bodily homeostatic control of fat mass is maintained. In contrast, during the ages of $50-60$, adipogenesis tends to dominate, suggesting an increase in adiposity in this age group. The process of adipogenesis occurs repeatedly throughout human life. In addition, AT turnover has been investigated, i.e., with the appearance of newly formed adipocytes, the oldest adipocytes die, are gradually removed from the tissue, and are replaced by new adipocytes. Changes in the number of adipocytes occur through a complex series of events involving proliferation and differentiation of preadipocytes. It is estimated that approximately $30 \%$ of the total cells present in AT are immature or less differentiated adipocytes and precursors or preadipocytes, i.e., cells that are capable of differentiating into mature adipocytes, assuming their specific physiological and morphological features.

The primary purpose of this review is to examine well established concepts in the literature as well as the current understanding of the metabolic (e.g., lipolysis, lipogenesis, and cell turnover) and endocrine actions of AT.

\section{Lipogenesis}

In adipocytes, triacylglycerols (TAGs), the major cellular constituents of white AT, are stored in a single large lipid inclusion or droplet. These droplets occupy 80$90 \%$ of the cell volume and the most central position of the cytoplasm, displacing the nucleus, cytosol, and remaining organelles to the periphery of the cell near the plasma cell membrane. Accumulation of TAGs can also occur in several other cell types; however, the occurrence of TAGs in other cell types is indicative of cellular malfunction and is a potential inductor of lipotoxic reactions that trigger cellular apoptosis. When such lipotoxicity occurs in muscle and liver, it leads to steatosis in addition to the development of insulin resistance, diabetes, and many other pathological diseases.

For the synthesis of TAGs, three moieties of fatty acids (FAs) are assembled with a molecule of glycerol. The AT is well prepared to perform this task; however, it requires sources of FAs and glycerol. FAs can be obtained through uptake from external sources [chylomicrons and very low-density lipoproteins (VLDLs)] or by local synthesis from acetyl coenzyme-A (CoA). Glycerol is obtained as glycerol3-phosphate (G3P) from glycolysis, glyceroneogenesis, and phosphorylation of glycerol by glycerol kinase activity.

\section{Lipogenic pathways}

\section{Sources of FAs}

FAs can be taken up as free FAs (FFAs), which are transported in the circulation bound to albumin or can be obtained by enzymatic hydrolysis of TAGs carried by lipoproteins such as chylomicrons (released from the intestinal tract after fat absorption) and, particularly, VLDLs, and to a lesser extent LDLs. The TAGs contained in these lipoproteins must first be hydrolyzed by lipoprotein lipase (LPL) located in the endothelium of the AT capillaries.

Adipocytes express several FA transporters located in the cell membrane to facilitate and control their transport, such as protein CD36, FA transporter protein, and membrane FA-binding protein (FABP). Because FAs are not soluble in the cytosolic aqueous medium and can induce toxic effects on membranes, once inside the cells, FAs bind to specific transporter proteins, known as FABPs (FABP-4 or aP2). These proteins carry FAs from the cell membrane to the site of action of the enzyme acyl-CoA synthase, whereby FAs are esterified with CoA to form acyl-CoA. Following this, acyl-CoA binds to and is carried by acyl-CoA-binding protein, to the sites of esterification with G3P to form TAG in the endoplasmic reticulum (ER).

\section{De novo lipogenesis}

De novo lipogenesis is the synthesis of FA from nonlipid substrates, primarily carbohydrates. De novo lipogenesis can occur both in the liver and in AT. The importance and contribution of the liver and AT varies between species. Studies indicate that de novo lipogenesis is less active in human AT than in the liver, on a per gram of tissue basis (4). Furthermore, controversy exists regarding differences in the lipogenic capacity of rats and humans. Although some studies indicate that this pathway is more active in rats, other studies suggest that such differences are due to diet composition, whereby when rats and humans consume diets with similar composition, a difference in lipogenic capacity is no longer observed (5).

For de novo lipogenesis to occur, acetyl-CoA is carboxylated by acetyl-CoA carboxylase (ACC) into malonyl-CoA, while oxaloacetate is reduced to malate by malate dehydrogenase $(\mathrm{MDH})$. Activation of FA synthase (FAS) allows malonyl-CoA and acetyl-CoA to assemble and to elongate the hydrocarbonic chain of FA, thus forming palmitic acid (16:0). Palmitic acid is then further elongated by an elongase to form stearic acid (18:0). Both palmitic acid and stearic acid are desaturated by stearoyl-CoA desaturase to form palmitoleic $(16: 1, n 7)$ and oleic acids $(18: 1, n 9)$, which are subsequently esterified with G3P to form TAG. 
An important cofactor in FA synthesis is reduced nicotinamide-adenine dinucleotide phosphate (NADPH), synthesized in the cytoplasm as a by-product of two pathways. The first pathway involves the following reactions: oxaloacetate formed by cleavage of cytosolic citrate is reduced to $\mathrm{MDH}$ by the enzyme NAD-malate dehydrogenase. This $\mathrm{MDH}$ then undergoes oxidative decarboxylation to form pyruvate and $\mathrm{CO}_{2}$, while generating NADPH from NADP ${ }^{+}$in a reaction catalyzed by malic enzyme. Reuptake of pyruvate by mitochondria occurs, whereby pyruvate combines with $\mathrm{CO}_{2}$ to regenerate oxaloacetate in a reaction catalyzed by the enzyme pyruvate carboxylase. The second pathway, or the pentose synthesis pathway, involves the conversion of glucose-6-phosphate (G6P) to 6-phosphogluconate by the action of G6P dehydrogenase (G6PDH).

It is suggested that, in primary white adipocyte culture, G6PDH mRNA levels change in parallel with FAS and ACC mRNA levels, indicating that this enzyme may be involved in the expression of other lipogenic enzymes (6). Accordingly, overexpression of G6PDH in mouse 3T3-L1 cells promoted the expression of adipogenic and lipogenic gene markers, including FAS, sterol regulatory-element binding protein 1c (SREBP-1c), peroxisome proliferatoractivated receptor-gamma (PPAR- $\gamma$ ), and aP2 (7).

\section{Production of G3P}

The synthesis of TAGs also requires a constant supply of G3P, as availability of G3P controls the esterification of FA. Hepatocytes use the glycerol released by AT during lipolysis for the phosphorylation of glycerol to G3P via the enzyme glycerol kinase (8). The presence of glycerol kinase in adipocytes is, however, controversial. In adipocytes, although some glycerol released during lipolysis can be directly phosphorylated and reused for TAG synthesis, the contribution of this pathway to G3P production is negligible. In contrast, studies have shown that G3P is generated in adipocytes via an important metabolic pathway known as glyceroneogenesis, which has been shown to be the quantitatively predominant source of G3P (9).

Hence, cytoplasmic G3P is derived from three pathways: glycolysis, gluconeogenesis, and glycerol kinase activity. In the glycolysis pathway, after entry into the cell, glucose is phosphorylated and ultimately converted via the glycolytic pathway to dihydroxyacetone phosphate (DHAP), and glyceraldehyde-3-phosphate. The DHAP is then further reduced by glycerol phosphate dehydrogenase (GPDH) to form G3P.

Through the glyceroneogenic pathway (which consists of the initial stages of canonical gluconeogenesis), precursors other than glycerol or glucose are converted to G3P, with the main substrates being pyruvate, lactate, and amino acids. Pyruvate is carboxylated to oxaloacetate, which then leaves the mitochondria and is decarboxylated by cytoplasmic phosphoenolpyruvate carboxykinase to form phosphoenolpyruvate. This is the rate-limiting step of the glyceroneogenic pathway (10). Phosphoenolpyruvate is then converted to glyceraldehyde-3-phosphate, which is reduced to DHAP by glyceraldehyde-3-phosphate dehydrogenase and then to G3P by GPDH.

\section{TAG synthesis}

In adipocytes, the biosynthesis of TAG is the result of esterification of alcoholic residues of G3P by various enzymes, namely, G3P acyltransferases (GPATs, the most abundant isoforms being GPAT1 and GPAT2), 1acylglycerol-3-phosphate acyltransferase (AGPAT, the most abundant isoform being AGPAT2), phosphatidic acid phosphatase, and diacylglycerol acyltransferase (DGAT, the most abundant isoforms being DGAT1 and DGAT2). The isoforms of these enzymes are encoded by different genes (11). All enzymes involved in this pathway of the biosynthesis of TAG are found in smooth ER. The importance of these enzymes in the control of TAG storage in AT has been demonstrated in studies of DGATdeficient mice and in studies of human subjects with congenital lipodystrophy (11).

A summary of all pathways involved in lipogenesis is provided in Figure 1.

\section{Regulation of lipogenesis}

\section{Nutritional}

Lipogenesis is highly influenced by factors such as feeding, fasting, and diet composition. Excessive carbohydrate consumption stimulates lipogenesis in both the liver and AT, increasing the availability of TAG in the postabsorptive state. In contrast, a high-fat/low-carbohydrate diet and fasting reduces de novo lipogenesis and lipogenesis, respectively, in AT (12). These changes are related to the increased or reduced expression and activity of LPL enzyme (high-carbohydrate/high-fat diets and fasting, respectively). In addition, it has been reported that the reduced lipogenic response during fasting is primarily due to a decreased capacity of white AT to generate acetyl-CoA from glucose, rather than an inhibition of lipogenic enzymes involved in FA synthesis.

Blood glucose levels act directly on lipogenic capability via three distinct mechanisms. First, because glucose is a substrate for lipogenesis, thus producing acetyl-CoA by glycolysis, glucose activates FA synthesis (13). Second, glucose stimulates the lipogenic enzyme synthesis of ATP-citrate lyase, G6PDH, ACC, malic enzyme, and FAS (8). Finally, glucose promotes lipogenesis by stimulating insulin secretion and inhibiting glucagon release from the pancreas (6).

\section{Hormonal}

Insulin is one of the most important hormonal factors that affect lipogenesis. Insulin increases glucose uptake in 


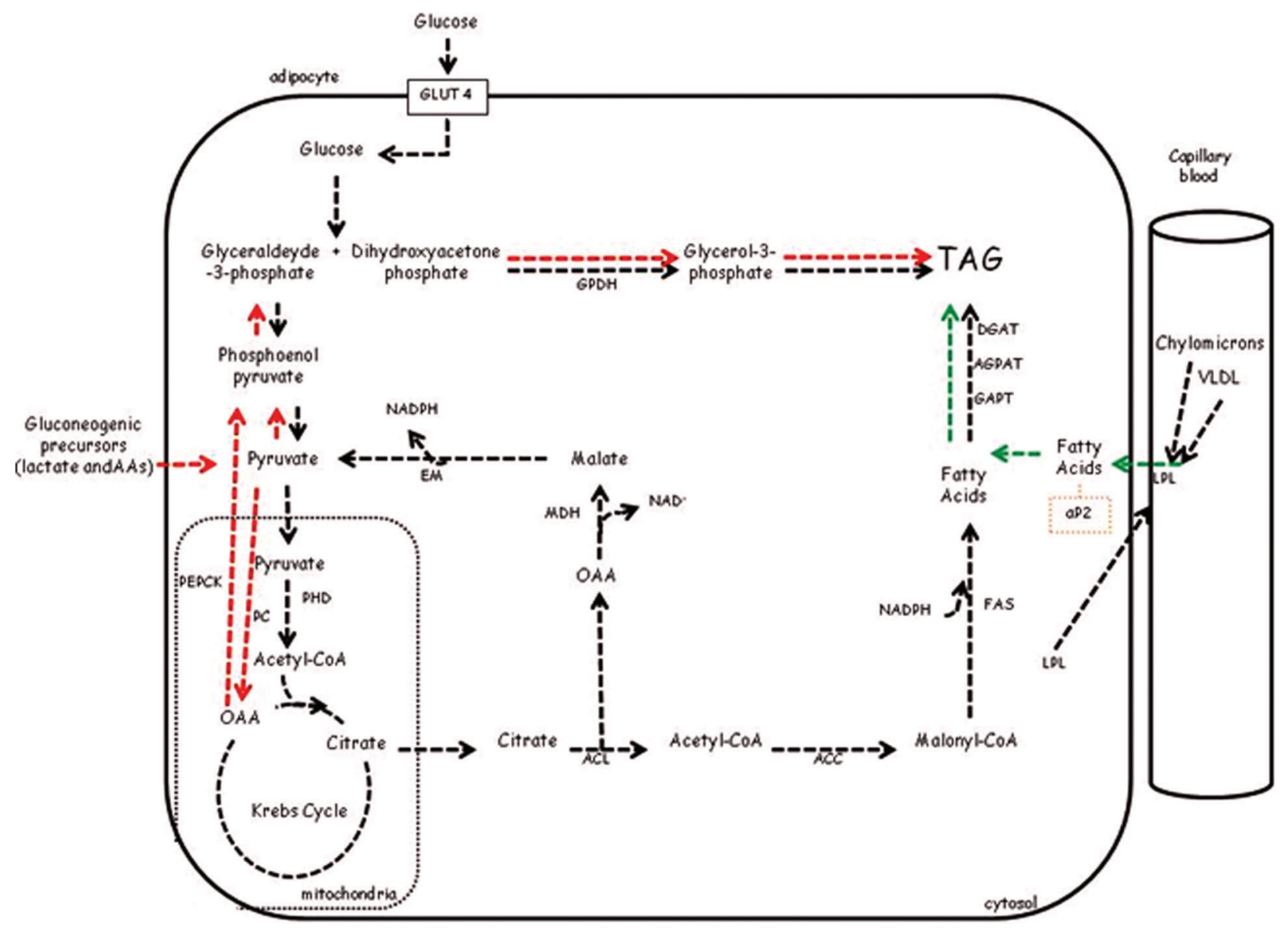

Figure 1. Lipogenic pathways. Black arrows: lipogenesis from glucose; red arrows: glyceroneogenesis; green arrows: TAG synthesis from circulating fatty acids. ACC: acetyl-CoA carboxylase; ACL: ATP-citrate lyase; AGPAT: 1-acylglycerol-3-phosphate acyltransferase; aP2: fatty acid binding protein; DGAT: diacylglycerol acyltransferase; ME: malic enzyme; FAS: fatty acid synthase; GPAT: glycerol-3-phosphate acyltransferase; GPDH: glycerol phosphate dehydrogenase; LPL: lipoprotein lipase; MDH: malate dehydrogenase; OAA: oxaloacetate; PC: piruvate carboxylase; PDH; piruvate dehydrogenase; PEPCK: phosphoenolpyruvate carboxykinase; VLDL: very low-density lipoprotein.

adipocytes via translocation of glucose transporters from the cytosol to the membrane and also activates lipogenic and glycolytic enzymes via covalent modification, thereby stimulating lipogenesis. Insulin also has effects on lipogenic gene expression (12), most likely via the transcription factor SREBP-1C. Furthermore, insulin increases expression of SREBP-1C, which induces expression and activity of the glucokinase enzyme in the liver, thereby increasing the concentration of G6P, which is reportedly the metabolite that mediates the effect of glucose on lipogenic gene expression. Conversely, it has been reported that glucagon lowers/inhibits lipogenesis by decreasing the activity of lipogenic enzymes (6).

Growth hormone also plays an important role in lipogenesis. Growth hormone dramatically reduces lipogenesis in AT, resulting in significant fat loss and a concomitant gain in muscle mass (14). Such metabolic side effects due to reduced lipogenesis in AT appear to be mediated by both a decrease in insulin sensitivity and a reduction in the number of insulin receptors, thus depressing gene expression of FAS enzyme and increasing the phosphorylation status of signal transducer and activator of transcription 5 (STAT5, $5 a$ and $5 b$ isoforms). The mechanism by which STAT5 diminishes lipid storage, however, remains unknown (15).

Leptin, a satiety hormone, expressed and secreted by adipocytes, acts on specific receptors located in both the brain and in peripheral regions of the body and may be involved in lipogenesis. It is well known that leptin limits lipid storage not only by inhibiting food intake, but also by affecting specific metabolic pathways in AT (16). Leptin induces the release of glycerol from adipocytes by stimulating FA oxidation and inhibiting the synthesis of FAs. This inhibition of FA synthesis by leptin is achieved 


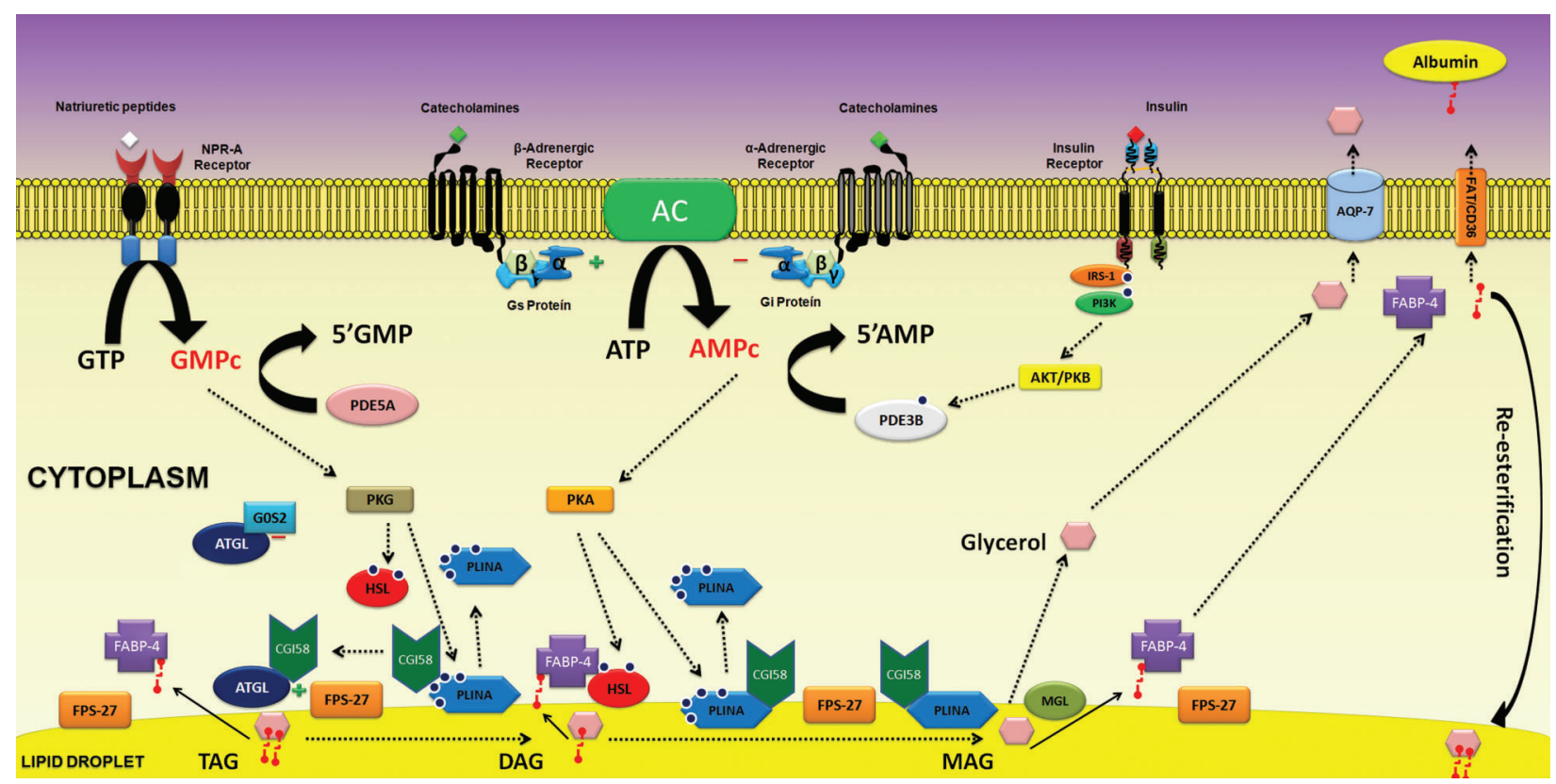

Figure 2. Major pathways involved in lipolytic regulation: the signal transduction pathways of catecholamines via adrenergic $[(\beta)$ stimulatory and $(\alpha 2)$ inhibitory] receptors and atrial natriuretic peptides via type A receptor (NPR-A); protein kinases (PKA and PKG) involved in the phosphorylation of target proteins; phosphorylation of HSL promoting translocation from cytosol to the surface of lipid droplets. Perilipin phosphorylation induces a major physical change on the droplet surface, which facilitates the action of HSL and starts lipolysis. Association of HSL with fatty acid binding protein (FABP-4) favors hydrolase action of HSL. Insulin anti-lipolytic action on adipocytes, through insulin receptors stimulation, leads to the activation of phosphodiesterase-3B (PDE-3B) promoting cAMP degradation. PDE-5A: phosphodiesterase 5A; ATGL: adipose tissue triacylglycerol lipase; FABP-4: fatty acid binding protein 4; GC: guanylate cyclase, Gi: inhibitory G protein; Gs: stimulatory G protein; HSL: hormone-sensitive lipase; PLINA: perilin; FPS-27: fatspecific protein 27; G0S2: G0/G1 switch gene 2; MGL: monoacylglycerol lipase; FFAs: free fatty acids; NPR-A: natriuretic peptide receptor-type A; TAG: triacylglycerol; DAG: diacylglycerol; MAG: monoacylglycerol.

by decreasing the expression of genes involved in FA and TAG synthesis (16). The transcription factor SREBP-1 is also inhibited by leptin, indicating that the inhibitory effect of leptin may also involve downregulation of the expression of lipogenic genes (17).

\section{Lipolysis: general mechanisms}

During periods of nutrient deprivation, stress, or physical exercise, lipolysis of TAG reserves is stimulated. Lipid droplets containing TAGs are surrounded by a phospholipid monolayer including structural proteins, enzymes, and coactivators. In general, lipolytic activity culminates in the systemic release of FFAs and glycerol. The release of FFAs into the blood is used as an energy source by other tissues, such as heart and skeletal muscle.

For the systemic release of FFAs and glycerol to occur, a number of extra- and intracellular events are required. These events include the presence of hormones that signal to adipocytes the need to release energy substrates to meet the increased demand of energy or to supply energy in cases of nutrient deprivation, thus sparing the use of glucose by lower priority body cells, because glucose is the primary energy source of the central nervous system.

\section{Messengers}

In humans, catecholamines, including epinephrine, norepinephrine, and insulin, are the primary regulators of lipolysis. It has also been reported in both in vivo and in vitro studies that natriuretic peptides (NPs), in addition to affecting cardiovascular and renal functions, are important stimulating agents for lipolysis.

The action of NPs occurs via activation of NP receptor A (NPR-A), which possesses intrinsic guanylate cyclase activity and thus enhances cyclic guanosine monophosphate (cGMP) levels, which, in turn, activate protein kinase $G(P K G)$, responsible for the phosphorylation and activation of hormone-sensitive lipase (HSL), an important TAG hydrolase (18). Moreover, the lipolytic effect promoted by NPs is unaffected by the primary anti-lipolytic action of insulin. This anti-lipolytic action is mediated through the activation of phosphodiesterase 3B (PDE3B), which degrades cyclic adenosine monophosphate (cAMP), the lipolytic mediator of the catecholamine pathway, but has no effect on cGMP (Figure 2). However, cGMP is degraded by another phosphodiesterase found in 
the adipocyte, PDE-5A. NPs emerged as potent regulators of lipolysis in humans, especially during exercise-stimulated lipolysis (18). Lipolytic effects stimulated by growth hormone, TNF- $\alpha$, adrenocorticotropin, and glucocorticoids have also been demonstrated (19-22).

Catecholamines remain the major lipolytic agents in adipocytes. Particularly in humans, during periods of fasting, catecholamines are the primary stimulators of lipolysis. The regulatory effects of catecholamines occur as a result of intracellular signaling triggered by activation of several adrenergic receptors, namely, $\beta-1, \beta-2, \beta-3$, and $\alpha-2$. In rodents, $\beta$-3-adrenergic receptor is the primary lipolytic route, whereas in humans, $\beta-1, \beta-2$, and $\alpha-2$ adrenergic receptors play a regulatory role (23). Adrenergic receptors have an extracellular and a transmembrane domain along with seven hydrophobic segments and an intracytoplasmic region that is coupled to regulatory GTP/GDP-associated proteins or $\mathrm{G}$ proteins (Figure 2). The ligand binding site of adrenergic receptors is located within the transmembrane domain. The $\beta$ adrenergic receptors are Gs-protein coupled receptors, which activate adenylate cyclase, a plasma membrane enzyme anchored to the inner cytoplasmic leaflet that catalyzes the formation of cAMP from ATP. In contrast, $\alpha$ 2-adrenergic receptors are Gi-protein coupled, inhibiting adenylate cyclase activation and thus preventing the formation of CAMP. Noradrenaline has a greater affinity for the $\alpha$-2-adrenergic receptor than for the $\beta$-adrenergic receptors, suggesting a role for the $\alpha$-adrenergic pathway in the regulation of lipolysis in human subcutaneous AT under conditions of exercise or stress (24).

The chemical messengers involved in the lipolytic activity of AT, such as catecholamines, insulin, NPs, and others cited earlier, are ultimately related to the synthesis or degradation of second messengers, such as CAMP and cGMP, which are involved in the activation of enzymes responsible for the control of lipolysis.

\section{Lipid droplets}

White adipocytes characteristically exhibit a large central fat droplet in the cytoplasm. In contrast, brown adipocytes show multiple small cytoplasmic fat droplets together with a rich amount of mitochondria, enabling these adipocytes a high capacity to oxidize substrates, primarily fatty acids. The lipid droplet is a vacuolar compartment responsible for the storage of neutral lipids. Movement of TAGs in and out of the lipid droplet is highly regulated (25). Monoacylglycerol (MAG) and diacylglycerol (DAG) molecules are intermediate compounds in the synthesis and degradation of TAGs. This lipid arrangement within the lipid droplet protects the adipocytes from harmful effects associated with excess intracellular fat, known as lipotoxicity. Consequently, disordered processing of fat that can potentially generate toxic fat metabolites, such as lipoperoxides, is avoided (25). Adipocyte hypertrophy and hyperplasia prevent accumulation of fat in the cytoplasm of other cell types in an organism unable to survive abnormal fat deposition. Indeed, the accumulation of abnormal fat may result in excess generation of ceramides, lipoperoxides, and other reactive oxygen and nitrogen species, such as nitric oxide and nitrolipids that trigger cellular apoptosis (26-28). In light of the above-mentioned studies, it may be assumed that lipid metabolism in adipocytes comprises mechanisms that control lipid mobilization via chemical messengers, with the lipid droplet as a target of all intracellular signaling.

\section{Enzymes, proteins, and coactivators}

Intracellular increases of cAMP and cGMP concentrations culminate in activation of dependent protein kinase A (PKA) and PKG, respectively. These enzymes transfer high-energy phosphate groups from donor molecules, such as ATP, to target proteins. The PKA enzyme consists of four subunits: two regulatory and two catalytic. In the presence of these activators, the regulatory subunit undergoes conformational changes resulting in catalytic activation.

Consequently, the $\beta$-adrenergic pathway, as described previously, promotes PKA activation. Once activated, PKA phosphorylates serine hydroxyl groups of HSL, resulting in activation of HSL (Figure 2). Once activated, HSL translocates from the cytosol to the lipid droplet, where it binds to FABP-4, ALBP, or aP2, and begins to hydrolyze TAGs, DAGs, and MAGs with relative hydrolytic rates of 1:10:0.5, respectively (29). Concurrently, PKA phosphorylates the lipid droplet surface protein perilipin, promoting displacement of perilipin to the cytosol. Such displacement of perilipin is in the opposite direction to the translocation of $\mathrm{HSL}$, allowing space in the lipid droplet interface for contact between the hydrolase and its substrate (30). Perilipin-1 is a member of the PAT lipid droplet protein family, which also includes adipophilin or adipocyte differentiation-related protein (currently named perilipin-2), a tail-interacting protein of $47 \mathrm{kDa}$ (currently named perilipin-3), S3-12 (currently named perilipin-4), OXPAT/MLDP (currently named perilipin-5), and fat-specific protein 27 (FSP27) (31). These proteins cover the lipid droplet surface, regulating and coordinating basal lipid storage and mediating stimulated lipolysis $(30,31)$. A study by Sztalryd et al. (32) showed that the presence and phosphorylation of perilipin-1 is essential for HSL translocation during lipolytic activity, as adipocytes isolated from mice that did not express perilipin-1 showed a loss of lipolytic activity following $\beta$-adrenergic stimulation. Although perilipin-1 restricts HSL access to TAG reserves, thereby reducing basal lipolysis, the phosphorylated form of perilipin-1 is essential for hydrolytic HSL activity during stimulated lipolysis.

In 2004, three independent groups reported the existence of a second enzyme involved in the hydrolysis of triglycerides, known as AT triglyceride lipase (ATGL), desnutrin, adiponutrin, or calcium-independent phospholipase $\mathrm{A} 2$. 
Consequently, the idea of HSL as the sole enzyme responsible for TAG hydrolysis in mammals was abandoned and replaced with the concept that HSL shares this ability equally with another enzyme (33). The ATGL enzyme is highly expressed in humans and rodents and has a high hydrolyzing activity on TAGs (33). In a study conducted by Haemmerle et al. (34) in 2006, ATGL knockout mice showed more than a $75 \%$ decrease in FFA release, with consequent accumulation of ectopic TAGs in muscle tissues, resulting in severe myopathy in cardiac muscle and a general weakness in energy balance, compromising animal survival.

The mechanisms that regulate activity of ATGL in response to $\beta$-adrenergic stimulation remain unknown. Although it is known that ATGL can be phosphorylated, it is unclear whether this modification is vital for ATGL activity (30). It is known, however, that ATGL activity is greatly enhanced in the presence of the coactivator comparative gene identification-58 (CGI-58), also known as $\alpha / \beta$-hydrolase domain-containing protein 5 . Reports suggest a mechanism involving perilipin-1 and CGI-58, whereby the availability of CGI-58 is dependent on perilipin-1 phosphorylation (Figure 2). In adipocytes with no lipolytic stimulation, CGI-58 is located on the lipid droplet surface next to perilipin-1, whereas, in the stimulated state, perilipin-1 is phosphorylated via PKA or PKG, resulting in displacement of perilipin-1 into the cytoplasm and dissociation of CGI-58, leaving CGI-58 freely available for interaction with and activation of ATGL (30). It has been reported that the presence of FSP27 on the lipid droplet surface exerts an important role in lipolytic activity of ATGL. Reduced expression of perilipin-1 and FSP27 on the lipid droplet surface results in elevated basal lipolysis (35). In contrast, when perilipin-1 and FSP27 work in unison on the lipid droplet surface, adipocyte lipolytic capacity is well maintained (36). Ultimately, FSP27 acts by limiting the presence of ATGL at the lipid droplet interface, whereas perilipin- 1 is crucial in control of the $\beta$-adrenergicmediated ATGL lipolytic response (36). To further increase the complexity of lipolysis, another protein, identified as G0/G1 switch gene 2, has been shown to interact with ATGL, inhibiting its activity (37). Furthermore, it has been shown that perilipin-5, expressed in both myocytes and brown adipocytes, can negatively modulate ATGL activity under basal conditions $(38,39)$. Such reports indicate that lypolytic action of ATGL is controlled by a variety of mechanisms, emphasizing the essential role of ATGL on lipid mobilization in adipocytes. Together, both ATGL and $\mathrm{HSL}$ are responsible for more than 95\% TAG hydrolysis in mouse adipocytes.

The other hydrolase located in adipocytes is MAG lipase (MGL). Unlike ATGL and HSL, MGL does not hydrolyze TAG and DAG, but it performs a specific action on MAG. Although its hydrolytic action is required for the complete hydrolysis of TAGs in vitro, the fact that HSL is also capable of hydrolyzing MAG indicates that the presence of MGL in vivo is not vital (30). MGL activity promotes dissociation of the last FA and glycerol of the MAG molecule. There is no evidence that cellular expression and activity of this enzyme are regulated by hormones or the energy state of the cell.

\section{Adipogenesis}

Adipogenesis is commonly known as the transformation of undifferentiated preadipocytes in AT to adipocytes. The balance between adipogenesis, triglyceride synthesis, and lipolysis is responsible for the quantity of AT in an organism. Consequently, knowledge of the steps involved in the regulation of adipogenesis is essential to understand the formation of AT. In addition, determination of the role of adipogenesis in metabolic conditions such as diabetes, obesity, and lipodystrophies may be important in the treatment of these diseases.

In obesity, the uncontrolled expansion of AT and dysregulation of AT function cause the clinical symptoms and comorbidities of this disease. Expansion of the AT mass, seen in obesity, involves both hyperplasia and hypertrophy of adipocytes. Thus, comprehension of the molecular basis of adipogenesis that it is responsible for hyperplasia and fat cell development in obesity would provide important information about new biomarkers and possible therapeutic targets for the development of antiobesity drugs.

Diabetes results in alterations in AT related to clinical features of this disorder. Type 2 diabetes is related to obesity and excess AT. In contrast, type 1 diabetes is characterized by a loss of AT mass. AT thus plays an important role in the maintenance of metabolic homeostasis. Therefore, as with obesity, research in adipogenesis may contribute to improvement in current treatments and development of new therapies for diabetes and obesity.

Adipogenesis comprises three distinct phases: growth arrest, clonal expansion, and terminal differentiation. These three stages are governed by four key transcription factors: the three CCAAT-binding proteins (C/EBPs) $\beta, \delta$, and $\alpha$ and PPAR- $\gamma$, expressed in a defined sequence and thus coordinating the series of adipogenic stages.

Although both C/EBP- $\beta$ and $\mathrm{C} / \mathrm{EBP}-\delta$ are expressed early in adipogenesis, they are not immediately active, allowing them to bind to the C/EBP-regulatory element (in the promoter region of $C / E B P-\alpha$ ) close to the beginning of clonal expansion (40). This delay in the ability of C/EBP- $\beta$ and $\mathrm{C} / \mathrm{EBP}-\delta$ to bind to gene promoter regions in in vitro studies differs from in vivo studies, whereby the onset of $C / E B P-\beta$ and $C / E B P-\delta$ expression coincides with their regulatory role on the C/EBP- $\alpha$ and PPAR- $\gamma$ promoter regions as well as on specific adipocyte genes (41). Expression of $C / E B P-\beta$ is required for the clonal expansion phase to occur (42). Increase in C/EBP- $\delta$ expression stimulates transcription of $C / E B P-\beta$, thus inducing expression of both C/EBP- $\alpha$ and PPAR- $\gamma$ (41). Both C/EBP- $\alpha$ and PPAR- $\gamma$ act on the promoter region of 
several specific adipocyte genes responsible for the adipocyte phenotype $(40,41)$.

In addition to these transcriptional factors, recent research has identified several mechanisms involved in the complex network that controls these adipogenic processes. Siersbaek et al. (43) demonstrated the important changes in chromatin structure in the adipogenesis process. During the first hours of adipogenesis, there is a significant modulation of the chromatin landscape that coincides with cooperative binding of multiple early transcriptional factors (including glucocorticoid receptor, retinoid $X$ receptor, Stat5a, C/EBP- $\beta$, and C/EBP- $\delta$ ). This binding enables chromatin remodeling and the binding of other transcriptional factors such as PPAR- $\gamma$.

Other important transcription factors include ZNF-638 and p204 protein. ZNF-638 belongs to the zinc finger family of proteins, is expressed in the early stages of adipogenesis, and physically interacts with $\mathrm{C} / \mathrm{EBP}-\beta$ and $C / E B P-\delta$ cooperating in the transcriptional stimulation of PPAR- $\gamma$ production (44). C/EBPs recruit ZNF-638 to the promoter region of PPAR- $\gamma$, indicating that this protein acts as a transcription cofactor (44).

Protein p204, which belongs to the interferon-inducible murine p200 protein family, is translocated to the nucleus in the early stages of adipogenesis, where it interacts with $\mathrm{C} / \mathrm{EBP}-\delta$, essential for its binding to the promoter region of PPAR- $\gamma(45)$.

Histone enzymatic modifications are also essential in adipogenesis (46), because they induce chromatin conformational changes required for the binding of transcriptional factors.

The histone lysine (K)-specific demethylase (KSD1) participates in the regulation of adipogenesis by demethylation of lysine 4 (K4) from histone H3 (H3K4) of the promoter region of $\mathrm{C} / \mathrm{EBP}-\alpha$, as well as other genes, enabling changes in the chromatin that allow access of transcriptional factors to the promoter regions (46).

Histone lysine-methyl-transferases are also involved in the regulation of adipogenesis. These enzymes provide gene silencing or activation by methylating histones into lysine residues (47).

Other signaling pathways involved in the control and development of adipogenesis is the MAP kinase (ERK1 and ERK2) pathway. Phosphorylation of ERK1 and ERK2 at specific sites is essential for the recruitment of preadipocytes and formation of mature adipocytes (48).

Therefore, adipogenesis involves the expression of key transcriptional factors (C/EBPs and PPAR- $\gamma$ ) vital for coordination of adipocyte differentiation, which is concurrently regulated by a large number of factors such as enzymes, proteins, and hormones, resulting in a complex but delicate regulatory system.

\section{Endocrine role of AT}

Initially considered an inert storage compartment for triglycerides, several studies have demonstrated that adipocytes are an abundant source of several proteins. The secretory function is an important feature of AT. Identification of leptin in 1994 (2) led to general recognition of AT as the owner of an important endocrine system responsible for synthesis and secretion of proteins (initially termed "adipocytokines" and currently known as adipokines) with biological activity involving not only adipocytes, but also other cells of the vascular stroma (49). A wide variety of these proteins has been and is still being identified, with the source attributed primarily to AT. Although adipokines have been the focus of much research in terms of their role as circulatory factors with effects on metabolically active tissues, it should be noted that adipocytes are responsive to various molecules secreted by other cells and tissues, e.g., the recently identified myokines (50). In the following section, the role of these adipokines in the regulation of different organs involved in cardiovascular, immune, reproductive, and metabolic systems is reviewed.

\section{Leptin}

Leptin, a $16-k D a$ protein hormone secreted primarily by AT, participates in the processes of growth regulation, metabolism, and behavior (especially feeding behavior). Leptin acts on the hypothalamus, modulating body weight, food intake, and lipid storage. Plasma levels of leptin correlate positively with body adiposity, and leptin secretion is many times higher in obese compared with lean subjects. Other tissues also express leptin, such as the placenta, mucosa of the gastric fundus, skeletal muscle, and mammary gland epithelial cells (51).

Leptin exerts its effect on several peripheral tissues by binding to its receptor, Ob-R, that belongs to Class 1 cytokine receptors as a member of the IL-6 family of receptors. The long isoform of the leptin receptor, Ob-Rb or Ob-RL, is found primarily in the brain, particularly in hypothalamic areas involved in the control of food intake. This receptor is also found in several peripheral tissues, including AT, placenta, adrenal medulla, liver, pancreatic beta cells, lung, intestinal cells, blood mononuclear cells, articular chondrocytes, heart, and skeletal muscle. The $O b-R$ gene also encodes an additional five short spliced forms of the leptin receptor (Ob-Ra, Ob-Rc, Ob-Rd, Ob$\mathrm{Re}$, and $\mathrm{Ob}-\mathrm{Rf}$ ) that are present in relatively low concentrations in the hypothalamus, microvessels, choroid plexus of the brain, as well as in all peripheral tissues (52).

In the hypothalamus, leptin signals the status of body energy reserves. When body energy reserves are plentiful, circulating levels of leptin are high, resulting in suppression of AMP-activated protein kinase (AMPK) activity in the medial hypothalamus (particularly in the arcuate nucleus), exerting anorexic effects that ultimately lead to weight loss. The inhibition of AMPK promotes activity of ACC in the arcuate and paraventricular nuclei of 
the hypothalamus (53). As a consequence, there is an increase in the level of malonyl-CoA, particularly in the arcuate nucleus, and an increase in the level of palmitoylCoA, particularly in the paraventricular nucleus, reducing the release of orexigenic peptides neuropeptide $Y$ and agouti-related protein, resulting in reduced food intake (53).

However, the effect of leptin on metabolism is not limited to the hypothalamus (51). Leptin acts directly on skeletal muscle increasing fatty acid oxidation via AMPK activation (54). Furthermore, it has been described in the literature that high plasma levels of leptin in obesity are related to insulin resistance. It has also been demonstrated that leptin reduces insulin sensitivity in isolated adipocytes and inhibits insulin secretion by pancreatic beta cells. In addition to metabolic effects, leptin has been recognized as playing an important role in modulating the immune system (55).

A decrease in the signaling or function of leptin receptors attenuates the inhibitory effect on food intake and reduces energy expenditure and leptin deficiency, ultimately resulting in severe obesity, hypogonadism, hyperinsulinemia, hyperphagia, and immune deficiency mediated by T-lymphocytes, which can be treated with hormonal replacement [for a review of leptin hormonal replacement, see Paz-Filho et al. (56)].

\section{Adiponectin}

Adiponectin was discovered in the 1990s by four independent groups, and it was originally named Acrp30, AdipoQ, apM1, and GBP28. Until recently, it was believed that adiponectin was secreted exclusively by AT; however, this has been challenged following demonstrations that this adipokine is also produced and secreted by murine and human cardiomyocytes and human and mouse skeletal muscle (57).

Adiponectin plasma levels are inversely proportional to body mass index and visceral adiposity and can be found in the circulation as a number of multimeric complexes: low molecular weight trimers, medium molecular weight hexamers, and high molecular weight (HMW) multimers (12 to 18 mers). The HMW multimer appears to be the most active form of adiponectin, as plasma concentration of HMW multimers is related to insulin sensitivity, and failure in the multimerization of adiponectin in humans is associated with type 2 diabetes mellitus (58).

Two types of adiponectin receptor have been described, AdipoR1 and AdipoR2. Depending on the type of receptor bound by adiponectin, a specific intracellular signaling pathway is activated: AMPK phosphorylation is predominant for AdipoR1, whereas AdipoR2 is involved in the activation of PPAR- $\alpha$ (59).

Adiponectin has several metabolic effects including anti-inflammatory, insulin sensitizing, anti-atherogenic (hypoadiponectemia is associated with a lipid profile favoring atherosclerosis), and hepatoprotective, preventing the development of non-alcohol-induced steatosis in ob/ob mice and LPL-induced liver damage in KK-Ay obese mice. In addition, it has been reported that adiponectin is involved in reduced risk of cardiovascular disease, inhibition of tumorigenesis, and increased production of IL-8 in human chondrocytes (57-59). Consequently, it is important to note that adiponectin is a promising therapeutic option for obesity-related diseases.

\section{IL-6}

IL-6 is involved in the pleiotropic effects implicated in the regulation of both inflammation and lipid metabolism. AT contributes approximately $35 \%$ of circulating IL-6, indicating that the IL- 6 released from AT may be associated with subclinical inflammatory states that result in insulin resistance. Indeed, in the liver IL-6 induces the activation of pathways that impair insulin action. Such effects of IL-6, however, are also apparently influenced by chronic IL-6 persistence, since during a physical activity the active muscles release IL- 6 that then acts as an activator of AMPK, stimulating glucose and fat burning in the tissue. In AT, it has been shown that IL-6 acts as a lipolytic agent $(60,61)$. In addition, research has demonstrated that a rise in IL-6 in the central nervous system is capable of reducing body weight and visceral adiposity without changing the amount of food ingested. Such body weight regulatory mechanisms involve sympathetic activity in brown AT followed by a more intense expression of mitochondrial uncoupling protein-1 (UCP-1), a likely enhancement of UCP-1, and a probable increase in thermogenesis (62). Finally, these data show that IL-6 has several metabolic effects that must be considered specific, depending on the site of action.

\section{TNF- $\alpha$}

The proinflammatory cytokine TNF- $\alpha$ induces both metabolic and immunological effects. Synthesized in both adipocytes and AT-infiltrated macrophages, TNF- $\alpha$ regulates the function and development of white AT by stimulating lipolysis and inhibiting lipogenesis and adipogenesis. Furthermore, TNF- $\alpha$ increases the expression of leptin while reducing adiponectin secretion. At the systemic level, TNF- $\alpha$ is known by its proinflammatory characteristics, which include reduction of insulin sensitivity in muscle, liver, and AT. Proposed TNF- $\alpha$ mechanisms for induction of insulin resistance include enhancement of FFA release into the circulation from lipolysis, decreased expression of glucose transporter type 4, and the harm to the insulin signaling pathways due to activation of serine protein kinases such as c-Jun kinase $(\mathrm{JNK})$ and $\mathrm{I} \kappa \mathrm{B}$ kinase that ultimately result in phosphorylation of serine residues of insulin receptor substrates IRS1 and IRS2 $(63,64)$.

\section{Resistin}

Resistin is expressed in adipocytes in rodents, 
whereas in humans it is synthesized by macrophages. Several tissues are responsive to resistin including AT, liver, muscle, vascular endothelium, and leukocytes. The main biological effects of resistin are associated with blood glucose homeostatic disturbances and increases in blood glucose levels in some animal models, partially explained as a consequence of increased hepatic glucose production. Furthermore, it has been reported that the absence of resistin restores hepatic insulin sensitivity, inhibiting the induction of hyperglycemia present in some animal models of obesity. Research has also shown that resistin reduces insulin-stimulated glucose uptake in isolated adipocytes. The mechanisms underlying these effects remain unclear, although data point to the suppression of AMPK activity by resistin, primarily in the liver, due to activation of the suppressor of cytokine stimulation-3 (SOCS3) (63). It also appears that resistin contributes to the pathogenesis of cardiovascular diseases such as atherosclerosis. High resistin levels are associated with elevated cardiovascular risk, unstable angina, endothelial dysfunction, rise in atherogenic proinflammatory markers, vascular smooth muscle cell proliferation, and unfavorable prognosis for coronary vascular disease. In human vascular endothelial cells, resistin augments the expression and secretion of endothelin-1, monocyte chemotactic protein-1 (MCP-1), and cell adhesion molecules such as ICAM-1 and VCAM1 and stimulates the migration and proliferation of these cells. Finally, it has been suggested that resistin can bind to certain endotoxin receptors, such as Toll-like receptor-4 (TLR4) (65).

\section{The renin-angiotensin-aldosterone system (RAAS)}

White AT cells, particularly white AT adipocytes, are capable of expressing all the RAAS components: angiotensinogen, renin, angiotensin-converting enzyme (ACE), angiotensin II receptors (AT1 and AT2), as well as the components of the nonclassical pathway including ACE2 and MAS receptors for angiotensin (1-7). Both metabolic and developmental processes in AT are regulated by RAAS. Both AT1 and AT2 receptors modulate AT mass expansion through an increase in lipogenesis (by AT2) and a reduction in lipolysis (by AT1). Therefore, both of these receptors have synergistic and additive effects on lipid storage in adipocytes. Associated with these, the RAAS produces an anti-adipogenic effect on human preadipocytes that assists in the expansion of the already hypertrophic adipose mass, resulting in inflammation and insulin resistance (66).

In 2012, it was reported that mature adipocytes are able to produce aldosterone. Aldosterone synthase mRNA (CYP11B2), as well as its resultant protein, were found in 3T3-L1 adipocytes and in mice and human mature adipocytes. Inhibition of CYP11B2 in 3T3-L1 cells decreased expression of key transcriptional factors related to adipogenesis, demonstrating a role for locally produced aldosterone on adipocyte differentiation (67). Recent research indicates that this system has a local decisive influence on the development of obesity-related hypertension (68).

\section{Vaspin}

Vaspin, of visceral adipose tissue-derived serpin, is a member of the serine protease inhibitor (serpin) family, which is highly expressed by visceral adipose tissue in obesity. It has been identified in an animal model of visceral adiposity and diabetes mellitus. Due to its almost exclusive expression in visceral AT, it was proposed that vaspin contributed to a compensatory mechanism in the pathogenesis of metabolic syndrome, as some vaspin agonists were able to improve glucose tolerance and insulin sensitivity (69). These effects were not confirmed, however, because vaspin expression increased with the development of insulin resistance in obesity (70). Regarding the effects on the cardiovascular system, low serum vaspin levels are assumed to be a predictor of coronary artery disease, although this statement remains controversial (71). As the physiological role of vaspin remains incomplete, with some studies suggesting an etiological participation and others proposing that vaspin is only a biomarker for inflammation and cardiovascular disease (72), further research is required to clarify the importance of vaspin in AT biology.

\section{Visfatin}

Visfatin was first recognized as a highly expressed adipokine in visceral AT; however, it is now known that expression of visfatin is far more ubiquitous, because it has been detected in many fat depots and other cell types. With a structure identical to two other molecules, pre-B cell colony-enhancing factor and nicotinamide phosphoribosyl-transferase (NAMPT), visfatin is capable of producing some insulin effects (via insulin receptor binding) in cell cultures and of diminishing blood glucose in mice, stimulating glucose uptake in cell culture and fat accumulation in preadipocytes (73-75).

It is important to note that visfatin has some catalytic properties resulting in functions similar to that of cytokineenzymes. Visfatin (or NAMPT) regulates intracellular activity of the NAD-consuming enzymes, stimulating the production of inflammatory cytokines and affecting the cell life span. Neutralizing the actions of visfatin brings benefits in models of inflammation, promoting hypotheses concerning the role of visfatin in metabolic and inflammatory diseases and in the development of atherosclerosis $(74,75)$.

\section{Omentin}

Omentin was so named because its main isoform is predominantly expressed in omental and epicardic fat, but not in subcutaneous depots. In vitro studies have shown that omentin is involved in improvement of glucose uptake 
and protein kinase B phosphorylation in human adipocytes and that expression of omentin in AT is reduced in obesity and insulin resistance (76). Rat arteries and human endothelial cells treated with omentin led to smooth muscle relaxation and to endothelial nitric oxide synthase (eNOS) phosphorylation and reduction of cyclooxygenase (COX)-2 expression and of TNF- $\alpha$ induced activation of the nuclear factor kappa-lightchain-enhancer of activated $B$ cells $(N F \kappa B)$ and JNKsignaling pathways in vascular endothelial cells, revealing its anti-inflammatory properties $(77,78)$.

\section{Apelin}

Apelin was first identified as an endogenous ligand of the G-protein-coupled apelin receptor APJ that activates intracellular pathways through the PI3K/AKT, ERK1/2, and P70-S6K pathways. In AT, apelin is synthesized and secreted by the adipocytes and vascular stromal cells. The main effects of apelin are related to body fluid homeostasis, such as control of thirst and diuresis, cardiovascular effects, including vasodilation via nitric oxide (NO) and opposing effects to the renin-angiotensin system, which includes the inhibition of angiotensin II signaling (79). Recently, it was demonstrated that lack of apelin increases susceptibility for post-ischemia cardiac lesions and that apelin analogs exhibit protective characteristics by promoting local angiogenesis (80). In terms of metabolism, apelin administration reduces body adiposity, improves glucose tolerance, and decreases insulin, TAG, and leptin serum concentrations in animal models of obesity. Apelin also appears to attenuate insulin resistance by increasing adiponectinemia, energy consumption, and the expression of mitochondrial UCPs in brown AT (81).

\section{Crosstalk between AT and other tissues}

In addition to the metabolic and endocrine functions of white adipocytes, white adipocytes express receptors for many molecules such as cytokines and hormones that exert autocrine, paracrine, and endocrine action. These molecules, which are released by several tissues and act on adipocytes, have the ability to modulate 1) endocrine function, regulating adipokine secretion; 2) cell number in the fat pad, regulating cell turnover (adipogenesis and apoptosis); and 3) metabolic regulation of lipogenesis, lipolysis, and oxidation $(10,82)$. Recent studies of metabolic function have focused on the relationship between the oxidative capacity control of white adipocytes and the regulation of metabolic homeostasis and body adiposity. This control is mediated by factors that regulate mitochondrial biogenesis and the expression of enzymes involved in thermogenesis, such as UCP-1 (50).

Recently, it has been reported that muscle is able to synthesize and secrete molecules known as myokines. These molecules are cytokines or other peptides that may act on different peripheral tissues such as liver, pancreas, blood vessels, bone, and AT (83). It has also been shown that the expression and secretion of these molecules is increased during exercise and that the stimulus for this increase is muscle contraction. The first myokine reported to be secreted into the bloodstream in response to muscle contractions was IL-6 (84), which is capable of inducing lipolysis in AT (85). Another protein that also has an important effect on AT is irisin, a fragment of a larger protein, fibronectin type III domain-containing protein 5 (FNDC5), which is expressed in skeletal muscle. Irisin, which is regulated by PPAR- $\gamma$ coactivator 1-alpha (PGC1$\alpha$ ), is secreted from muscle into blood (in both mouse and human) and activates thermogenic functions in AT, increasing the expression of UCP-1 in mitochondria, as well as the density of mitochondria in white adipocytes. Irisin increases energy expenditure likely through stimulation of UCP-1 and brown-fat-like development and has been found to improve glucose tolerance in obese animals (84). Thus, this crosstalk between AT and muscle is a promising area of research in the study of new therapeutic approaches for metabolic disorders such as obesity and diabetes.

\section{Final considerations}

This review reports the impressive amount of research regarding AT biology. Currently, there remains a strong tendency to classify AT as an organ. As AT is diffusely distributed throughout the body, and as the metabolic and endocrine functions of AT vary depending on the anatomical localization of the depot, a new approach concerning the paracrine effects of AT is currently gaining importance in recent research. Furthermore, as the differences in AT are so specific to the tissue location, many researchers are now considering the existence of various adipose organs in the body. Another branch of adipose research is related to brown AT. It is now widely accepted that brown fat is not only functional but also widely distributed in adult humans. As brown adipocytes are diffusely and dispersedly distributed within the fat pads, particularly in the subcutaneous depots, and the amount of brown fat is decreased in obesity, attempts to increase brown fat are important goals in future therapeutic strategies to deal with obesity and the associated complications. Therefore, understanding the functional abilities of AT and the potential physiological and pathophysiological roles of AT will bring new and fundamental therapeutic tools to treat the obesity epidemic and related morbidities.

\section{Acknowledgments}

Research supported by FAPESP (\#2009/54732-7), CNPq, and CAPES. 


\section{References}

1. Fonseca-Alaniz MH, Takada J, Alonso-Vale MI, Lima FB. [The adipose tissue as a regulatory center of the metabolism]. Arq Bras Endocrinol Metabol 2006; 50: 216-229, doi: 10.1590/S0004-27302006000200008.

2. Zhang $Y$, Proenca R, Maffei M, Barone M, Leopold L, Friedman JM. Positional cloning of the mouse obese gene and its human homologue. Nature 1994; 372: 425-432, doi: 10.1038/372425a0.

3. Ahima RS. Adipose tissue as an endocrine organ. Obesity 2006; 14 (Suppl 5): 242S-249S, doi: 10.1038/oby.2006.317.

4. Diraison $F$, Dusserre $E$, Vidal $H$, Sothier $M$, Beylot $M$. Increased hepatic lipogenesis but decreased expression of lipogenic gene in adipose tissue in human obesity. $A m \mathrm{~J}$ Physiol Endocrinol Metab 2002; 282: E46-E51.

5. Swierczynski J, Goyke E, Wach L, Pankiewicz A, Kochan Z, Adamonis $W$, et al. Comparative study of the lipogenic potential of human and rat adipose tissue. Metabolism 2000; 49: 594-599, doi: 10.1016/S0026-0495(00)80033-5.

6. Girard J, Perdereau D, Foufelle F, Prip-Buus C, Ferre P. Regulation of lipogenic enzyme gene expression by nutrients and hormones. FASEB J 1994; 8: 36-42.

7. Park J, Rho HK, Kim KH, Choe SS, Lee YS, Kim JB. Overexpression of glucose-6-phosphate dehydrogenase is associated with lipid dysregulation and insulin resistance in obesity. Mol Cell Biol 2005; 25: 5146-5157, doi: 10.1128/ MCB.25.12.5146-5157.2005.

8. Coleman RA, Lee DP. Enzymes of triacylglycerol synthesis and their regulation. Prog Lipid Res 2004; 43: 134-176, doi: 10.1016/S0163-7827(03)00051-1.

9. Nye CK, Hanson RW, Kalhan SC. Glyceroneogenesis is the dominant pathway for triglyceride glycerol synthesis in vivo in the rat. J Biol Chem 2008; 283: 27565-27574, doi: 10.1074/jbc.M804393200.

10. Olswang $\mathrm{Y}$, Cohen $\mathrm{H}$, Papo $\mathrm{O}$, Cassuto $\mathrm{H}$, Croniger $\mathrm{CM}$, Hakimi $P$, et al. A mutation in the peroxisome proliferatoractivated receptor gamma-binding site in the gene for the cytosolic form of phosphoenolpyruvate carboxykinase reduces adipose tissue size and fat content in mice. Proc Natl Acad Sci U S A 2002; 99: 625-630, doi: 10.1073/ pnas.022616299.

11. Agarwal AK, Garg A. Congenital generalized lipodystrophy: significance of triglyceride biosynthetic pathways. Trends Endocrinol Metab 2003; 14: 214-221, doi: 10.1016/S10432760(03)00078-X.

12. Wong RH, Sul HS. Insulin signaling in fatty acid and fat synthesis: a transcriptional perspective. Curr Opin Pharmacol 2010; 10: 684-691, doi: 10.1016/j.coph.2010.08. 004.

13. Haugen F, Drevon CA. The interplay between nutrients and the adipose tissue. Proc Nutr Soc 2007; 66: 171-182, doi: 10.1017/S0029665107005423.

14. Borland CA, Barber MC, Travers MT, Vernon RG. Inhibition of adipose tissue lipogenesis by growth hormone: role of polyamines. Biochem Soc Trans 1993; 21: 400S.

15. Rosenfeld RG, Hwa V. The growth hormone cascade and its role in mammalian growth. Horm Res 2009; 71 (Suppl 2): 36-40, doi: 10.1159/000192434.

16. Oswal A, Yeo G. Leptin and the control of body weight: a review of its diverse central targets, signaling mechanisms, and role in the pathogenesis of obesity. Obesity 2010; 18 : 221-229, doi: 10.1038/oby.2009.228.

17. Nogalska A, Sucajtys-Szulc E, Swierczynski J. Leptin decreases lipogenic enzyme gene expression through modification of SREBP-1c gene expression in white adipose tissue of aging rats. Metabolism 2005; 54: 1041-1047, doi: 10.1016/j.metabol.2005.03.007.

18. Lafontan M, Moro C, Berlan M, Crampes F, Sengenes C, Galitzky J. Control of lipolysis by natriuretic peptides and cyclic GMP. Trends Endocrinol Metab 2008; 19: 130-137, doi: 10.1016/j.tem.2007.11.006.

19. Gravholt CH, Schmitz O, Simonsen L, Bulow J, Christiansen JS, Moller N. Effects of a physiological GH pulse on interstitial glycerol in abdominal and femoral adipose tissue. Am J Physiol 1999; 277: E848-E854.

20. Gasic S, Tian B, Green A. Tumor necrosis factor alpha stimulates lipolysis in adipocytes by decreasing $\mathrm{Gi}$ protein concentrations. J Biol Chem 1999; 274: 6770-6775, doi: 10.1074/jbc.274.10.6770.

21. Kiwaki K, Levine JA. Differential effects of adrenocorticotropic hormone on human and mouse adipose tissue. $J$ Comp Physiol B 2003; 173: 675-678, doi: 10.1007/s00360003-0377-1.

22. Campbell JE, Peckett AJ, D'souza AM, Hawke TJ, Riddell MC. Adipogenic and lipolytic effects of chronic glucocorticoid exposure. Am J Physiol Cell Physiol 2011; 300: C198C209, doi: 10.1152/ajpcell.00045.2010.

23. Langin D. Adipose tissue lipolysis as a metabolic pathway to define pharmacological strategies against obesity and the metabolic syndrome. Pharmacol Res 2006; 53: 482-491, doi: 10.1016/j.phrs.2006.03.009.

24. de Glisezinski I, Larrouy D, Bajzova M, Koppo K, Polak J, Berlan $\mathrm{M}$, et al. Adrenaline but not noradrenaline is a determinant of exercise-induced lipid mobilization in human subcutaneous adipose tissue. J Physiol 2009; 587: 33933404, doi: 10.1113/jphysiol.2009.168906.

25. Greenberg AS, Coleman RA, Kraemer FB, McManaman JL, Obin MS, Puri V, et al. The role of lipid droplets in metabolic disease in rodents and humans. J Clin Invest 2011; 121: 2102-2110, doi: 10.1172/JCI46069.

26. Unger RH, Zhou YT. Lipotoxicity of beta-cells in obesity and in other causes of fatty acid spillover. Diabetes 2001; 50 (Suppl 1): S118-S121, doi: 10.2337/diabetes.50.2007.S118.

27. Boland MP, O'Neill LA. Ceramide activates NFkappaB by inducing the processing of p105. J Biol Chem 1998; 273: 15494-15500, doi: 10.1074/jbc.273.25.15494.

28. Lin KT, Xue JY, Nomen M, Spur B, Wong PY. Peroxynitriteinduced apoptosis in HL-60 cells. J Biol Chem 1995; 270: 16487-16490, doi: 10.1074/jbc.270.28.16487.

29. Lampidonis AD, Rogdakis E, Voutsinas GE, Stravopodis DJ. The resurgence of Hormone-Sensitive Lipase (HSL) in mammalian lipolysis. Gene 2011; 477: 1-11, doi: 10.1016/ j.gene.2011.01.007.

30. Lass A, Zimmermann R, Oberer M, Zechner R. Lipolysis - a highly regulated multi-enzyme complex mediates the catabolism of cellular fat stores. Prog Lipid Res 2011; 50: 14-27, doi: 10.1016/j.plipres.2010.10.004.

31. Xu L, Zhou L, Li P. CIDE proteins and lipid metabolism. Arterioscler Thromb Vasc Biol 2012; 32: 1094-1098, doi: 
10.1161/ATVBAHA 111.241489.

32. Sztalryd C, Xu G, Dorward H, Tansey JT, Contreras JA, Kimmel AR, et al. Perilipin A is essential for the translocation of hormone-sensitive lipase during lipolytic activation. J Cell Biol 2003; 161: 1093-1103, doi: 10.1083/jcb.200210169.

33. Zimmermann R, Strauss JG, Haemmerle G, Schoiswohl G, Birner-Gruenberger R, Riederer M, et al. Fat mobilization in adipose tissue is promoted by adipose triglyceride lipase. Science 2004; 306: 1383-1386, doi: 10.1126/science.1100 747.

34. Haemmerle G, Lass A, Zimmermann R, Gorkiewicz G, Meyer C, Rozman J, et al. Defective lipolysis and altered energy metabolism in mice lacking adipose triglyceride lipase. Science 2006; 312: 734-737, doi: 10.1126/science. 1123965.

35. Nishino N, Tamori $\mathrm{Y}$, Tateya S, Kawaguchi T, Shibakusa T, Mizunoya W, et al. FSP27 contributes to efficient energy storage in murine white adipocytes by promoting the formation of unilocular lipid droplets. J Clin Invest 2008; 118: 2808-2821.

36. Yang X, Heckmann BL, Zhang X, Smas CM, Liu J. Distinct mechanisms regulate ATGL-mediated adipocyte lipolysis by lipid droplet coat proteins. Mol Endocrinol 2013; 27: 116126, doi: 10.1210/me.2012-1178.

37. Yang X, Lu X, Lombes M, Rha GB, Chi YI, Guerin TM, et al. The $G(0) / G(1)$ switch gene 2 regulates adipose lipolysis through association with adipose triglyceride lipase. Cell Metab 2010; 11: 194-205, doi: 10.1016/j.cmet.2010.02.003.

38. Brasaemle DL. Perilipin 5: putting the brakes on lipolysis. J Lipid Res 2013; 54: 876-877, doi: 10.1194/jlr.E036962.

39. Wang H, Bell M, Sreenivasan U, Hu H, Liu J, Dalen K, et al. Unique regulation of adipose triglyceride lipase (ATGL) by perilipin 5, a lipid droplet-associated protein. J Biol Chem 2011; 286: 15707-15715, doi: 10.1074/jbc.M110.207779.

40. Tang QQ, Lane MD. Activation and centromeric localization of CCAAT/enhancer-binding proteins during the mitotic clonal expansion of adipocyte differentiation. Genes Dev 1999; 13: 2231-2241, doi: 10.1101/gad.13.17.2231.

41. Salma N, Xiao H, Imbalzano AN. Temporal recruitment of CCAAT/enhancer-binding proteins to early and late adipogenic promoters in vivo. $\mathrm{J} \mathrm{Mol}$ Endocrinol 2006; 36: 139151, doi: 10.1677/jme.1.01918.

42. Tang $Q Q$, Otto TC, Lane MD. CCAAT/enhancer-binding protein beta is required for mitotic clonal expansion during adipogenesis. Proc Natl Acad Sci U S A 2003; 100: 850855, doi: 10.1073/pnas.0337434100.

43. Siersbaek R, Nielsen R, John $S$, Sung MH, Baek S, Loft A, et al. Extensive chromatin remodelling and establishment of transcription factor 'hotspots' during early adipogenesis. EMBO J 2011; 30: 1459-1472, doi: 10.1038/emboj.2011.65.

44. Meruvu S, Hugendubler L, Mueller E. Regulation of adipocyte differentiation by the zinc finger protein ZNF638. J Biol Chem 2011; 286: 26516-26523, doi: 10.1074/jbc. M110.212506.

45. Xiao J, Sun B, Cai GP. Transient expression of interferoninducible p204 in the early stage is required for adipogenesis in 3T3-L1 cells. Endocrinology 2010; 151: 3141-3153, doi: 10.1210/en.2009-1381.

46. Musri MM, Carmona MC, Hanzu FA, Kaliman P, Gomis R, Parrizas M. Histone demethylase LSD1 regulates adipogenesis. J Biol Chem 2010; 285: 30034-30041, doi: 10.1074/
jbc.M110.151209.

47. Okamura M, Inagaki T, Tanaka T, Sakai J. Role of histone methylation and demethylation in adipogenesis and obesity. Organogenesis 2010; 6: 24-32, doi: 10.4161/org.6.1.11121.

48. Donzelli E, Lucchini C, Ballarini E, Scuteri A, Carini F, Tredici G, et al. ERK1 and ERK2 are involved in recruitment and maturation of human mesenchymal stem cells induced to adipogenic differentiation. $\mathrm{J} \mathrm{Mol} \mathrm{Cell} \mathrm{Biol} \mathrm{2011;} \mathrm{3:} \mathrm{123-}$ 131, doi: 10.1093/jmcb/mjq050.

49. Funahashi $T$, Nakamura $T$, Shimomura I, Maeda $K$ Kuriyama $\mathrm{H}$, Takahashi $\mathrm{M}$, et al. Role of adipocytokines on the pathogenesis of atherosclerosis in visceral obesity. Intern Med 1999; 38: 202-206, doi: 10.2169/internalmedicine. 38.202.

50. Pedersen BK, Febbraio MA. Muscles, exercise and obesity: skeletal muscle as a secretory organ. Nat Rev Endocrinol 2012; 8: 457-465, doi: 10.1038/nrendo.2012.49.

51. Fried SK, Ricci MR, Russell CD, Laferrere B. Regulation of leptin production in humans. J Nutr 2000; 130: 3127S$3131 \mathrm{~S}$

52. Tartaglia LA. The leptin receptor. J Biol Chem 1997; 272: 6093-6096

53. Gao S, Kinzig KP, Aja S, Scott KA, Keung W, Kelly S, et al Leptin activates hypothalamic acetyl-CoA carboxylase to inhibit food intake. Proc Natl Acad Sci U S A 2007; 104 17358-17363, doi: 10.1073/pnas.0708385104.

54. Minokoshi Y, Kim YB, Peroni OD, Fryer LG, Muller C, Carling $D$, et al. Leptin stimulates fatty-acid oxidation by activating AMP-activated protein kinase. Nature 2002; 415 339-343, doi: 10.1038/415339a.

55. Schaffler A, Scholmerich J, Salzberger B. Adipose tissue as an immunological organ: Toll-like receptors, C1q/TNFs and CTRPs. Trends Immunol 2007; 28: 393-399, doi: 10.1016/ j.it.2007.07.003.

56. Paz-Filho G, Wong ML, Licinio J. Ten years of leptin replacement therapy. Obes Rev 2011; 12: e315-e323, doi: 10.1111/j.1467-789X.2010.00840.x.

57. Brochu-Gaudreau K, Rehfeldt C, Blouin R, Bordignon V, Murphy BD, Palin MF. Adiponectin action from head to toe. Endocrine 2010; 37: 11-32, doi: 10.1007/s12020-009-92788.

58. Waki H, Yamauchi T, Kamon J, Ito Y, Uchida S, Kita S, et al. Impaired multimerization of human adiponectin mutants associated with diabetes. Molecular structure and multimer formation of adiponectin. J Biol Chem 2003; 278: 4035240363, doi: 10.1074/jbc.M300365200.

59. Lee MH, Klein RL, El-Shewy HM, Luttrell DK, Luttrell LM The adiponectin receptors AdipoR1 and AdipoR2 activate ERK1/2 through a Src/Ras-dependent pathway and stimulate cell growth. Biochemistry 2008; 47: 11682-11692, doi: 10.1021/bi801451f.

60. Kim JH, Bachmann RA, Chen J. Interleukin-6 and insulin resistance. Vitam Horm 2009; 80: 613-633, doi: 10.1016/ S0083-6729(08)00621-3.

61. Hoene $M$, Weigert $C$. The role of interleukin- 6 in insulin resistance, body fat distribution and energy balance. Obes Rev 2008; 9: 20-29.

62. Li G, Klein RL, Matheny M, King MA, Meyer EM, Scarpace PJ. Induction of uncoupling protein 1 by central interleukin- 6 gene delivery is dependent on sympathetic innervation of brown adipose tissue and underlies one mechanism of body 
weight reduction in rats. Neuroscience 2002; 115: 879-889, doi: 10.1016/S0306-4522(02)00447-5.

63. Galic S, Oakhill JS, Steinberg GR. Adipose tissue as an endocrine organ. Mol Cell Endocrinol 2010; 316: 129-139, doi: 10.1016/j.mce.2009.08.018.

64. Cai D, Yuan M, Frantz DF, Melendez PA, Hansen L, Lee J, et al. Local and systemic insulin resistance resulting from hepatic activation of IKK-beta and NF-kappaB. Nat Med 2005; 11: 183-190, doi: 10.1038/nm1166.

65. Schwartz DR, Lazar MA. Human resistin: found in translation from mouse to man. Trends Endocrinol Metab 2011; 22: 259-265.

66. Yvan-Charvet L, Quignard-Boulange A. Role of adipose tissue renin-angiotensin system in metabolic and inflammatory diseases associated with obesity. Kidney Int 2011; 79: 162-168, doi: 10.1038/ki.2010.391.

67. Briones AM, Nguyen Dinh CA, Callera GE, Yogi A, Burger $\mathrm{D}, \mathrm{He} \mathrm{Y}$, et al. Adipocytes produce aldosterone through calcineurin-dependent signaling pathways: implications in diabetes mellitus-associated obesity and vascular dysfunction. Hypertension 2012; 59: 1069-1078, doi: 10.1161/ HYPERTENSIONAHA.111.190223.

68. Yiannikouris F, Gupte M, Putnam K, Thatcher S, Charnigo $\mathrm{R}$, Rateri $\mathrm{DL}$, et al. Adipocyte deficiency of angiotensinogen prevents obesity-induced hypertension in male mice. Hypertension 2012; 60: 1524-1530, doi: 10.1161/HYPERTE NSIONAHA.112.192690.

69. Wada J. Vaspin: a novel serpin with insulin-sensitizing effects. Expert Opin Investig Drugs 2008; 17: 327-333, doi: 10.1517/13543784.17.3.327.

70. Shaker OG, Sadik NA. Vaspin gene in rat adipose tissue: relation to obesity-induced insulin resistance. Mol Cell Biochem 2013; 373: 229-239, doi: 10.1007/s11010-0121494-5.

71. Kobat MA, Celik A, Balin M, Altas Y, Baydas A, Bulut M, et al. The investigation of serum vaspin level in atherosclerotic coronary artery disease. J Clin Med Res 2012; 4: 110-113.

72. Wang Z, Nakayama T. Inflammation, a link between obesity and cardiovascular disease. Mediators Inflamm 2010; 2010: 535918, doi: 10.1155/2010/535918.

73. Fukuhara A, Matsuda M, Nishizawa M, Segawa K, Tanaka $\mathrm{M}$, Kishimoto K, et al. Visfatin: a protein secreted by visceral fat that mimics the effects of insulin. Science 2005; 307: 426-430, doi: 10.1126/science.1097243.

74. Romacho T, Sanchez-Ferrer CF, Peiro C. Visfatin/Nampt: an adipokine with cardiovascular impact. Mediators Inflamm 2013; 2013: 946427, doi: 10.1155/2013/946427.

75. Moschen AR, Gerner RR, Tilg H. Pre-B cell colony enhancing factor/NAMPT/visfatin in inflammation and obesity-related disorders. Curr Pharm Des 2010; 16: 19131920, doi: 10.2174/138161210791208947.

76. de Souza Batista CM, Yang RZ, Lee MJ, Glynn NM, Yu DZ, Pray J, et al. Omentin plasma levels and gene expression are decreased in obesity. Diabetes 2007; 56: 1655-1661, doi: $10.2337 / \mathrm{db06}-1506$.

77. Yamawaki H, Kuramoto J, Kameshima S, Usui T, Okada M, Hara Y. Omentin, a novel adipocytokine inhibits TNFinduced vascular inflammation in human endothelial cells. Biochem Biophys Res Commun 2011; 408: 339-343, doi: 10.1016/j.bbrc.2011.04.039.

78. Yamawaki H, Tsubaki N, Mukohda M, Okada M, Hara Y. Omentin, a novel adipokine, induces vasodilation in rat isolated blood vessels. Biochem Biophys Res Commun 2010; 393: 668-672, doi: 10.1016/j.bbrc.2010.02.053.

79. Chun HJ, Ali ZA, Kojima Y, Kundu RK, Sheikh AY, Agrawal $\mathrm{R}$, et al. Apelin signaling antagonizes Ang II effects in mouse models of atherosclerosis. J Clin Invest 2008; 118: 3343-3354.

80. Wang W, McKinnie SM, Patel VB, Haddad G, Wang Z, Zhabyeyev $P$, et al. Loss of Apelin exacerbates myocardial infarction adverse remodeling and ischemia-reperfusion injury: therapeutic potential of synthetic Apelin analogues. J Am Heart Assoc 2013; 2: e000249.

81. Higuchi K, Masaki T, Gotoh K, Chiba S, Katsuragi I, Tanaka $\mathrm{K}$, et al. Apelin, an APJ receptor ligand, regulates body adiposity and favors the messenger ribonucleic acid expression of uncoupling proteins in mice. Endocrinology 2007; 148: 2690-2697, doi: 10.1210/en.2006-1270.

82. Jensen MD, Ekberg K, Landau BR. Lipid metabolism during fasting. Am J Physiol Endocrinol Metab 2001; 281: E789E793.

83. Bostrom P, Wu J, Jedrychowski MP, Korde A, Ye L, Lo JC, et al. A PGC1-alpha-dependent myokine that drives brownfat-like development of white fat and thermogenesis. Nature 2012; 481: 463-468, doi: 10.1038/nature10777.

84. Pedersen BK, Febbraio MA. Muscle as an endocrine organ: focus on muscle-derived interleukin-6. Physiol Rev 2008; 88: 1379-1406, doi: 10.1152/physrev.90100.2007.

85. Van Snick J. Interleukin-6: an overview. Annu Rev Immunol 1990; 8: 253-278, doi: 10.1146/annurev.iy.08.040190. 001345. 